\title{
Agôn
}

Revue des arts de la scène

HS 1 | 2011

Mettre en scène l'événement

\section{« Le duo des dieux » : rhétoriques et montage des discours Bush / Ben Laden dans 11 septembre 2001 de Michel Vinaver}

Marianne Noujaim

\section{(2) OpenEdition \\ Journals}

Édition électronique

URL : http://journals.openedition.org/agon/1766

DOI : 10.4000/agon. 1766

ISSN : 1961-8581

Éditeur

Association Agôn

Référence électronique

Marianne Noujaim, « «Le duo des dieux » : rhétoriques et montage des discours Bush / Ben Laden dans 11 septembre 2001 de Michel Vinaver », Agôn [En ligne], HS 1 | 2011, mis en ligne le 19 août 2011, consulté le 17 avril 2020. URL : http://journals.openedition.org/agon/1766 ; DOI : https://doi.org/ 10.4000/agon. 1766 


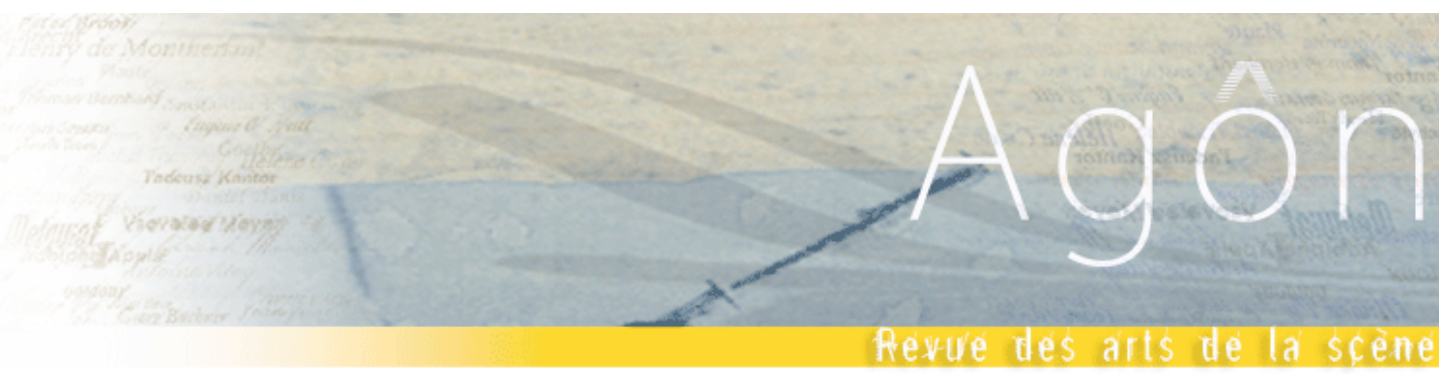

\section{«Le duo des dieux " : rhétoriques et montage des discours Bush / Ben Laden dans 11 septembre 2001 de Michel Vinaver}

\section{Marianne NOUJAIM}

Le 11 septembre 2001 étant, comme le note Michel Vinaver, « un événement que la pensée ne réussit pas à circonscrire ${ }^{1} »$, il pose un défi à la représentation, notamment discursive. D'ailleurs, de par sa nature, l'acte marque un retour à la violence primitive, pardelà la convention de la verbalisation des conflits qui assoit le règne de la rhétorique en politique. Or, c'est une profusion chaotique d'images, mais aussi de discours, qui déferle dans la couverture médiatique de l'événement. Cherchant à le fixer dans l'ampleur de sa fulgurance, avant que la mémoire s'applique à la tamiser, Michel Vinaver prélève dans la presse et brasse dans le texte de 11 septembre $2001^{2}$ des fragments des discours prononcés par les «grands» et les «petits», les responsables, les acteurs, les témoins et victimes de l'événement et d'autres bribes de discours journalistiques qu'il fait énoncer par le chœur et par le journaliste-narrateur, dans une forme qu'il compare à celle de l'oratorio ${ }^{3}$.

Cette pièce où se combinent lyrisme et narration se clôt par une confrontation dramatique entre les deux discours que Bush et Ben Laden prononcent et font diffuser le 7 octobre 2001. Or, ces deux discours témoignent d'un retour au régime de la rhétorique performative cautionnant les actes violents à entreprendre ou entrepris. Pour marquer l'enjeu de ces deux pouvoirs qui s'affrontent discursivement et se répondent tout en feignant de s'ignorer, Michel Vinaver choisit « la construction dramatique et la structure arbitraire d'un dialogue improbable [qui] fait voler en éclats la surface des mots et des choses pour donner

\footnotetext{
${ }^{1}$ Michel Vinaver, « Notes manuscrites pour 11 septembre 2001 », in Lexi / textes 9 - Inédits et commentaires,

${ }^{2}$ Michel Vinaver, 11 septembre 2001, in Théâtre complet 8, Paris, L’Arche, 2002.

${ }^{3}$ Michel Vinaver, « Note liminaire », in 11 septembre 2001, op. cit., p. 133.
} 
accès à la vraie violence du politique ${ }^{4} »$. Nous examinerons la manière dont le montage dialogal opéré par l'auteur fait ressortir avec plus de force les travers et paradoxes des rhétoriques somme toute similaires adoptées par chacun des deux responsables ${ }^{5}$, et observerons comment il exacerbe la charge à la fois ironique et tragique de ce finale constitué d'un « duo des dieux ${ }^{6} »$.

\section{Rhétoriques spéculaires et performativité de la parole monologique}

Alors même que l'on a considéré que les événements du 11 septembre confirment la thèse de Huntington sur le Choc des civilisations, il apparaît dans la pièce qu'il s'agit bel et bien d'un dialogue et d'une osmose, aussi flagrants qu'involontaires, de modes de parler et d'agir :

Bush

Bonjour sur mes ordres l'armée des États-Unis a commencé des frappes

Ben Laden

Voici l'Amérique frappée par Dieu tout puissant en un de ses organes vitaux

Bush

Nous sommes soutenus dans cette opération par la volonté collective du monde entier

\section{Ben Laden}

De sorte que ses plus grands édifices sont pulvérisés par la grâce de Dieu à qui va notre gratitude $[\ldots]$

Bush

Et nous sommes les amis de près d'un milliard de gens qui pratiquent la religion islamique dans le monde entier

Ben Laden

L'hypocrisie a levé haut la tête lamentant la perte de ces assassins qui ont joué avec le sang l'honneur et les sacrements des musulmans

Bush

Nous sommes une nation pacifique

\footnotetext{
${ }^{4}$ Catherine Naugrette, Paysages dévastés. Le théâtre et le sens de l'humain, Paris, Circé, «Penser le théâtre », 2004, p. 58.

${ }^{5} C f$. l'analyse détaillée des similitudes et divergences des mécanismes rhétoriques des deux discours par Bruce Lincoln, "Symmetric Dualism : Bush and bin Laden on October 7", Holy Terrors : Thinking About Religion after September 11, University of Chicago Press, 2002, pp. 19-32. Également disponible en ligne : http://www.press.uchicago.edu/Misc/Chicago/481921.html [Site consulté le 05/07/11].

${ }^{6}$ Michel Vinaver, « Notes manuscrites pour 11 septembre 2001 », op. cit., p. 283.
} 
Ben Laden

Ils ont soutenu le boucher contre sa victime l'oppresseur contre l'enfant

innocent

Bush

Face à la nouvelle menace d'aujourd'hui la seule façon de poursuivre la paix

Ben Laden

Le vent du changement souffle

Bush

Est de poursuivre ceux qui la menacent

Ben Laden

Pour faire disparaître le mal

Bush

Le nom de l'opération militaire d'aujourd'hui

Ben Laden

Je jure devant Dieu

Bush

Est Liberté Immuable [...]

La paix et la liberté l'emporteront

Ben Laden

Que Dieu nous protège

Bush

Que Dieu nous bénisse ${ }^{7}$

L'impact déstabilisateur de l'acte terroriste se révèle dans la mesure où l'illogisme de ce dernier se répercute, à travers les mimétismes discursifs et argumentatifs, sur les langages économiques et politiques du camp visé, dans une sorte de fascination vertigineuse qui souligne la précarité de ce camp ${ }^{8}$. Si l'on considère les deux premières répliques, on note d'emblée le bouclage lexical et sémantique qui les lie ( « frappes » / « frappée » avec inversion des agents et des patients des «frappes ») ; de même la série de répliques « La seule façon de poursuivre la paix / Le vent du changement souffle / Est de poursuivre ceux qui la menacent / Pour faire disparaître le mal »présente un enchaînement syntaxique et sémantique parfaitement cohérent des répliques des deux locuteurs ; enfin, les deux répliques finales

\footnotetext{
${ }^{7}$ Michel Vinaver, 11 septembre 2001, op. cit., pp. 173-181.

${ }^{8}$ À ce sujet, Michel Vinaver confie à J.-L. Rivière lors d'un entretien : «J'ai été très frappé quand j'ai vu que l'éditorial d'un tout récent numéro du International Herald Tribune avait pour titre "We have some planes". C'est une citation extraite du prérapport de la commission d'enquête parlementaire américaine sur le 11 septembre. Il s'agit d'un propos qui est attribué à Mohammed Atta - on n'en est pas tout à fait sûr, mais il a été capté dans les minutes précédant l'attentat - et c'est la première réplique de 11 septembre 2001 : "We have some planes". » (Michel Vinaver et Jean-Loup Rivière, « Le théâtre comme objet fractal », in Michel Vinaver, Europe, $\mathrm{n}^{\circ} 924,2006$, p. 131.)
} 
attestent le parallélisme rythmique, syntaxique et sémantique des deux énoncés qui se répondent et se répètent. Sur le plan des mécanismes argumentatifs également, les deux discours se font écho. Ben Laden invoque Dieu comme auteur de l'acte perpétué ; et Bush se dote des attributs d'omnipotence, d'omniprésence et d'omniscience'. Ben Laden s'associe avec ses partisans, par analogie, à l'« enfant innocent» persécuté par des «bourreaux », et accuse les victimes de l'attentat d'être des assassins; et Bush parvient, par un paralogisme tirant parti de la polysémie du verbe « poursuivre », à tirer la conclusion de la nécessité de la guerre à partir d'une prémisse assurant le pacifisme américain (« la seule façon de poursuivre la paix est de poursuivre ceux qui la menacent »).

C'est que le discours extrémiste ou totalitariste, comme l'écrit Bertrand Buffon dans La Parole persuasive (2002), « ne procède pas par enchaînements dûment justifiés de propositions mais par juxtaposition d'énoncés dont la seule proximité est censée valoir lien logique. [En effet,] les multitudes ne sont jamais impressionnées par la logique d'un discours, mais bien par les images sentimentales que certains mots et associations de mots font naître ${ }^{10} »$. Fondés sur les raisonnements sophistiques comme le paradoxe et sur le culte de l'éthos (ou de l'image oratoire du locuteur, en l'occurrence déifié), les discours extrémistes tirent leurs ressources non point des finesses de la persuasion mais de leur capacité à subjuguer l'auditoire par l'autorité de la parole que nous qualifierons, en référence à Bakhtine, de «monologique ». En effet, l'exclusion idéologique se traduit par la division manichéenne du monde fondant le discours de Ben Laden, qui oppose «les infidèles, les hypocrites, ces assassins qui ont joué avec le sang l'honneur et les sacrements des musulmans, le boucher, l'oppresseur, le mal, l'armée des infidèles, le camp des mécréants », au « camp des croyants » et «à un groupe de musulmans d'élite» auxquels «Dieu a confié [...] la mission sacrée de détruire l'Amérique / Que Dieu leur accorde une place suprême dans les cieux ${ }^{11}$. Cette dichotomie s'impose comme un impératif et le discours, qui échappe aux recours de la raison et de la discussion, interdit toute réponse ou réciprocité ${ }^{12}$.

\footnotetext{
${ }^{9}$ Bruce Lincoln met à jour l'inscription dans les deux discours, de manière explicite chez Ben Laden et sur le mode de la connotation et du sous-texte chez Bush, des lieux communs et images coraniques ou bibliques.

${ }^{10}$ Bertrand Buffon, La Parole persuasive. Théorie et pratique de l'argumentation rhétorique, Paris, PUF, «L'interrogation philosophique », 2002, p. 388.

${ }^{11}$ Michel Vinaver, 11 septembre 2001, op. cit., p. 175.

${ }^{12}$ Voir Daniel Weiss, "Stalinist vs. Fascist propaganda. How much do they have in common?" in Louis de Saussure and Peter Schulz (eds), Manipulation and Ideologies in the Twentieth Century, Amsterdam / Philadelphia, John Benjamins Publishing Company, 2005, pp. 251-274. L'auteur dégage les similitudes syntaxiques et sémantiques des deux discours totalitaires fondés, entre autres procédés, sur l'axiologisation manichéenne du monde et des valeurs, appuyée par le processus syntaxique de l'adjonction au substantif de
} 
En contrepartie, le discours de Bush est centré autour de l'idée - somme toute, inattendue et quelque peu inappropriée dans ces circonstances-là - de la «Liberté Immuable », symbole national américain, invoqué pour justifier les décisions prises et « tous les sacrifices à venir », et qui donne son nom à l'opération militaire déclenchée. Les valeurs de «la paix », de «la justice», de « la liberté », de «la générosité de l'Amérique » lui apportent alors le soutien et « la volonté collective du monde entier » dans sa guerre contre «les terroristes [qui] auront beau s'enfoncer au plus profond de leurs cachettes souterraines $»^{13}$. On voit comment la rhétorique des idéologies extrémistes ajuste la réalité à leur propre vision, à leur propre logos qui cautionne et camoufle le caractère irraisonnable des actions entreprises ${ }^{14}$. Les faits, les images des locuteurs eux-mêmes, de leurs ennemis et les croyances des auditeurs sont ainsi soumis à un processus de schématisation langagière par les « chefs » qui agissent sur le réel en le déformant et le transformant selon leurs propres finalités. La performativité du langage l'emporte dans ce duo final sur sa fonction référentielle. De fait, les deux discours regorgent d'actes de langage et de performatifs. Bush déclare la guerre et Ben Laden riposte par un appel au combat adressé à tout Musulman et par la menace lancée contre «l'hypocrisie», soit contre les États-Unis et leurs alliés : «La bataille est maintenant engagée », « Je jure devant Dieu [...] Que l’Amérique ne vivra pas en paix $\gg{ }^{15}$.

\section{Montage ironique et tragique contemporain}

Or, ces mécanismes de la rhétorique extrémiste sont mis à mal par le montage déconstructeur opéré par l'auteur. Le parallélisme entre les deux discours, qui sera souvent établi par d'autres que Michel Vinaver, est mis ici à jour sans que l'auteur rajoute de son cru

\footnotetext{
superlatifs, d'adjectifs ou d'adverbes intensificateurs ou modificateurs, autant de procédés que l'on observe dans les deux discours cités dans 11 septembre 2001.

${ }^{13}$ Michel Vinaver, 11 septembre 2001, op. cit., p. 175.

${ }^{14}$ Par suite, « la parole n'est plus envisagée sous l'angle de la raison mais sous celui de la force. [...] La parole est considérée comme un son, une onde dont la perception doit déclencher des images, des émotions et des actions. Les mots sont conçus comme des flèches ou des balles. La question n'est pas de les faire accepter consciemment mais de les implanter dans le cerveau de l'auditeur. [...] La langue est transformée pour empêcher toute prise de conscience du mensonge idéologique et rendre la contestation impensable. Effacement du réel et manipulation du langage donnent au raisonnement un caractère infalsifiable. » (Bertrand Buffon, La Parole persuasive, op. cit., p. 382, p. 385).

${ }^{15}$ Michel Vinaver, 11 septembre 2001, op. cit., p. 179.
} 
aux citations qu'il découpe et monte uniquement. Il semble en effet que le message de la pièce, jugée «tendancieuse », apparaît d'autant plus subversif et dérangeant que Michel Vinaver n'a rien écrit lui-même dans ce passage. Il s'agit là d'un flagrant exemple des effets de la polyphonie vinavérienne dont l'ironie dérange les classifications manichéennes du monde et les monologismes idéologiques et religieux, sans verser ni dans le militantisme ni dans la profession de foi.

Toutefois, sans être pure négativité du renvoi dos à dos des deux discours, le montage ironique permet d'appréhender l'impensé et la complexité du fait terroriste ainsi que l'illogisme des rhétoriques monologiques qui s'y rattachent ou en découlent. À ce titre, Sébastien Rongier voit dans la forme moderne de l'ironie une «inconciliation» avec l'absolutisme des formes (rationnelles ou, dans ce cas, irrationnelles) de la domination :

\begin{abstract}
L'incertitude ironique n'est pas un discours de savoir mais une prise de conscience dans son mouvement d'un événement complexe. Elle se heurte à la mise en ordre par une expérience de l'impensé. L'impensé du discours renverse les neutralisations rhétoriques et retrouve une part étymologique du substantif discours. Car discours, du latin discursus, substantif dérivé du verbe discurrere, signifie 'courir ça et là'. L'impensé du discours, c'est le ça et là d'une course rendue folle par les inflexions systématisantes et absolutistes de la raison [...]. En déjouant les formes d'organisation stables, l'ironie retrouve une capacité critique par un mouvement oblique. [...] Le processus ironique, rompant avec les catégories, pénètre dans les interstices et fragmente les discours pour en explorer le ça et là. L'ironie peut engendrer une certaine équivocité mais pas une incohérence. [...] Car l'inconciliation ironique est le travail souterrain d'une pensée qui affronte les formes de la domination. La disjonction ironique comme enjeu de la modernité s'exprime sur le mode du fragmentaire et du montage, de l'inachèvement ou de l'aporie ${ }^{16}$.
\end{abstract}

Par la place qu'il accorde à la parole et au témoignage des victimes, des simples individus et du chœur, le théâtre vinavérien affirme que la seule position admise est celle qui consiste à s'orienter « du côté du petit contre le grand, du faible contre le fort ${ }^{17}$ ». Ce faisant, il renoue aussi avec une tradition du théâtre tragique antique qu'il réadapte en fonction de l'actualité. Ce ne sont plus des rois ni des reines qui révoquent un ordre politique, social ou divin, mais des individus qui malgré eux peut-être accusent, par leurs paroles, l'existence encore de nos jours de systèmes asservissants. Le chœur exprime alors, à travers la souffrance collective, les mesquineries et les échecs des supra-structures qui se disloquent et déraisonnent à l'instar des actes et des discours des terroristes. La fonction du théâtre dans 11

\footnotetext{
16 Sébastien Rongier, De l'ironie. Enjeux critiques pour la modernité, Paris, Klincksieck, «Collection d'esthétique », 2007, pp. 89-92.

${ }^{17}$ Michel Vinaver et Fabienne Darge, « Michel Vinaver, dramaturge du réel », propos recueillis par Fabienne Darge, Le Monde 2, n 258, 24 au 30 janvier 2009, p. 25.
} 
septembre 2001 n'est pas sans rappeler celle que Michel Vinaver lui-même avait déjà soulignée en 1957, à propos de l'adaptation en français des parties chorales de l'Antigone de Sophocle, pièce qu'il considère à plusieurs égards contemporaine :

\begin{abstract}
Le mouvement de la pièce ne se réduit pas, loin de là, à une bascule entre deux discours constitués qui s'opposent. Le mouvement profond, sous-jacent, de l'œuvre, est celui d'une agonie collective irréversible, historique, celle de la Cité de Thèbes. [...] La Cité est souillée et les dieux semblent ne plus s'y intéresser, sont insupportablement absents. Antigone et Créon tenteront - chacun suivant son tempérament et ses valeurs - d'engager les dieux dans l'histoire [...], d'engager les dieux à leur côté et de rejeter sur l'adversaire la responsabilité de l'apocalypse... [...] Le chœur, témoin de tous les dialogues et de tous les conflits, semble percevoir par instants, au-delà des affrontements quotidiens, cette seconde et invisible tragédie où les personnages s'opposent par leur folie plus que par leur raison... et s'unissent dans la folie ${ }^{18}$.
\end{abstract}

En réalité, l'ironie culmine dans la pièce dans la mesure où, au « duo des dieux » formant la parole des « grands », succède la réplique finale de la pièce énoncée par une jeune femme rescapée de l'effondrement des tours grâce à l' " indigestion » et au « vomi[ssement] » nocturnes de son fils qui lui ont occasionné " une mine de déterrée », l'empêchant ainsi d'assister au «meeting de clôture» de son entreprise dans lequel « une opération assez faramineuse » de «sept milliards de dollars en actions et trois et demi comptant plus quatre milliards huit cent mille de dettes » devait se conclure ${ }^{19}$. Les sentiments d' " inachèvement » et d'《aporie » ironiques suscités par la prise de conscience de la catastrophe par la jeune femme se traduisent dans les derniers mots de la pièce «Et maintenant et maintenant et maintenant $»^{20}$, mots qui résonnent de manière tout aussi ironique et tragique pour le spectateur-lecteur contemporain, qui assiste encore aujourd'hui aux fatales conséquences de ce « duo des dieux ».

En somme, 11 septembre 2001 de Vinaver souligne à la fois la contradiction et l'osmose, dans les discours tenus au lendemain de l'événement éponyme, entre le grand et le petit, le bien et le mal, l'horreur et l'ordinaire, pointant par l'ironie du montage la forme

\footnotetext{
${ }^{18}$ Cf. Michel Vinaver, «Pour un théâtre des idées » (1982), in Écrits sur le théâtre 2, Paris, L’Arche, 1998, p. 13.

${ }^{19}$ Michel Vinaver, 11 septembre 2001, op. cit., p. 181.

${ }^{20} I d$.
} 
contemporaine du tragique. Par-delà les discours qui s'affichent comme antagonistes mais usent de stratégies parallèles d'endoctrinement, l'auteur donne à entendre, à travers les rescapés et le chœur, le témoignage de ceux que les systèmes tentent de cloisonner dans leurs visions. Pièce sur les conséquences les plus catastrophiques de l'échec du dialogue et des pouvoirs des paroles monologiques, 11 septembre 2001 montre qu'en définitive, tous les excès se ressemblent et contribuent à asservir l'individu moderne, indirectement invité à se démarquer de tout endoctrinement politique ou idéologique.

À propos de l'auteur :

Marianne Noujaïm est titulaire d'un doctorat en études théâtrales (cotutelle Paris 3 / USEK). Sa thèse porte sur le dialogisme et la polyphonie dans le théâtre de Michel Vinaver. Elle est actuellement professeur assistant a l'Université Saint-Esprit de Kaslik (Liban).

Pour citer ce document :

Marianne Noujaim, «« Le duo des dieux » : rhétoriques et montage des discours Bush / Ben Laden dans 11 septembre 2001 de Michel Vinaver», Agôn [En ligne], Dossiers, HS n ${ }^{\circ} 1$ : Mettre en scène l'événement, 11 septembre 2001 de Michel Vinaver, mis à jour le : 05/09/2011, URL : http://agon.ens-lyon.fr/index.php?id=1766. 